\title{
Evaluation of the Water Quality of River Kaduna, Nigeria Using Water Quality Index
}

\author{
${ }^{* 1}$ OGBOZIGE, FJ; ${ }^{1}$ ADIE, DB; ${ }^{1}$ IGBORO, SB; ${ }^{2}$ GIWA, A \\ ${ }^{1}$ Department of Water Resources and Environmental Engineering, Ahmadu Bello University, Zaria \\ 2 Departments of Polymer and Textile Science, Ahmadu Bello University, Zaria \\ Corresponding author: engr.ogbozige@gmail.com (+2349037494999)
}

\begin{abstract}
Twelve (12) water quality parameters (turbidity, TDS, pH, $\mathrm{Cl}^{-}, \mathrm{EC}, \mathrm{DO}, \mathrm{BOD}_{5}$, COD, total nitrogen, total phosphorus, Fe and $\mathrm{Mn}$ ) were analyzed in River Kaduna, Nigeria on a monthly basis for a period of one year in 15 sampling locations using standard methods. The data obtained were used to develop Water Quality Index (WQI) across the 15 sampling locations. The WQI revealed that the water quality of 4 sampling locations were poor as their index values ranged between 17.77 to 25.47 . On the other hand, the generalized water quality of the remaining 11 sampling locations was marginal as the index values ranged between 44.95 to 60.80 . The index values of the various sampling locations were thereafter used as weights in mapping the WQI of the entire sampled portion of the river using Inverse Distance Weighted (IDW) interpolation method. The WQI of the entire river was suggestively ranked marginal as 11 sampling locations out of $15(73.3 \%)$ fell into the marginal category. Hence, regulatory agencies were advised to check the anthropogenic activities along the watershed with more emphasis at the hot spot areas or locations that recorded poor WQI. C) JASEM
\end{abstract}

https://dx.doi.org/10.4314/jasem.v21i6.21

Key Words: WQI, Canadian, Mapping, Sampling, Locations.

Proper documentation of the water quality in a given catchment is important because it will suggest the level of treatment to be given to the water when the need for using such water for a particular purpose arises. This is because the cost of treating raw water per unit volume is a function of the quality status of the raw water. Therefore, a strategic means of cutting down the cost of treatment of raw water is to manage the pollution load of the rivers serving as source of raw water.

An integral part in any environmental monitoring program is the reporting of results to both managers and the general public. However, most water quality researchers report results by comparing the different analyzed parameters with their respective permissible limits set by regulating bodies (local or international). For instance, over the years, several researchers such as Mohammed et al. (2015), Mohammed (2013) and Yusuf et al. (2008) have reported the water quality of River Kaduna by describing the trends and compliance with official stated guidelines. However, Carlos and Alejandra (2014) stated that in many cases, managers and the general public rather prefer statements concerning the general health or status of the system concern. Hence, the Canadian Council of Ministers of Environment (2001) reported that one possible solution to this problem is by employing an index that will mathematically combine all water quality measures and provide a general and readily understood description of the water. In other words, developing Water Quality Index (WQI) for River Kaduna will summarize the various analyzed water ingredients (parameters) and rank the overall quality of the water. The ranking could be excellent, good, fair, marginal or poor.

\section{MATERIALS AND METHOD}

Description of Study Area: River Kaduna is a tributary of the River Niger with its source from Kujama Hill in Plateau State and flow for $210 \mathrm{~km}$ before reaching Kaduna town. It crosses the city dividing it into north and south areas. Beyond Kaduna, the river flows for about $100 \mathrm{~km}$ into the Shiroro Dam. It continues to flow for $100 \mathrm{~km}$ and finally discharges into River Niger at the northern shores of Pategi (Ekiye and Luo, 2010). However, the portion of the river considered was $32.7 \mathrm{~km}$ stretch that cut across four Local Government Areas of Kaduna State which are parts of Igabi, Kaduna North, Chikun and Kaduna South (Figure 1). This stretch of the river falls between Latitudes $10^{\circ} 2800^{\prime \prime}-10^{\circ} 36^{\prime}$ $00^{\prime \prime}$ North and longitude $07^{\circ} 21^{\prime} 00^{\prime \prime}-07^{\circ} 35^{\prime} 00^{\prime \prime}$ East (ArcGIS 10.5).

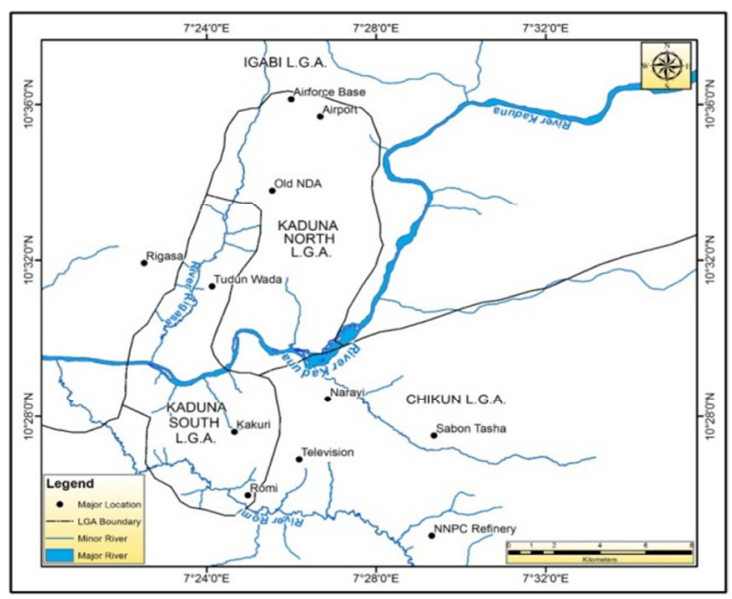


Fig 1: Map of River Kaduna within Kaduna metropolis.

Sampling Locations: The sampling locations comprises of 8 along River Kaduna and 7 (at about $30 \mathrm{~m}$ away from the confluence points) along the major tributaries, making a total of 15 sampling sites. These stations correspond to flow routs and inflow from discharge point. The justification for selecting these locations as sampling points is that, they represented the best point for gaining access to the rivers and also suitable for easy sampling of the current water quality status and have a more progressive pollution load (Adie, 2008).

At each sampling location, a Global Position System (GPS) was used in recording the geographical coordinate of such location. The recorded coordinate of all the sampling locations are shown in Table 1

Table 1: Sampling location coordinates

\begin{tabular}{|c|c|c|}
\hline $\begin{array}{c}\text { Location } \\
\text { Code }\end{array}$ & Location Name & Geographical Coordinate \\
\hline L1 & Malali & $10^{\circ} 36^{\prime} 3.09^{\prime \prime} \mathrm{N}, 7^{\circ} 30^{\prime} 21.91 " \mathrm{E}$ \\
\hline L2 & Kwarau & $10^{\circ} 36^{\prime} 16.96^{\prime \prime N}, 7^{\circ} 30^{\prime} 5.43^{\prime \prime E}$ \\
\hline $\mathbf{L 3}$ & NNPC & $10^{\circ} 31 ' 29.23 " \mathrm{~N}, 7^{\circ} 28^{\prime} 14.04 " \mathrm{E}$ \\
\hline L4 & Kuyi & $10^{\circ} 30^{\prime} 56.02^{\prime \prime} \mathrm{N}, 7^{\circ} 28^{\prime} 28.84^{\prime \prime} \mathrm{E}$ \\
\hline L5 & Barnawa & $10^{\circ} 29^{\prime} 44.46 " \mathrm{~N}, 7^{\circ} 26^{\prime} 56.86^{\prime \prime} \mathrm{E}$ \\
\hline L6 & Kutimbi & $10^{\circ} 28^{\prime} 53.12^{\prime \prime} \mathrm{N}, 7^{\circ} 27^{\prime} 6.71^{\prime \prime} \mathrm{E}$ \\
\hline L7 & Living Faith & $10^{\circ} 29^{\prime} 36.82^{\prime \prime} \mathrm{N}, 7^{\circ} 26^{\prime} 16.25^{\prime \prime} \mathrm{E}$ \\
\hline L8 & Kigo & $10^{\circ} 29^{\prime} 57.44^{\prime \prime} \mathrm{N}, 7^{\circ} 26^{\prime} 3.32^{\prime \prime} \mathrm{E}$ \\
\hline L9 & Down Quarters & $10^{\circ} 29^{\prime} 6.80^{\prime \prime} \mathrm{N}, 7^{\circ} 24^{\prime} 13.53 " \mathrm{E}$ \\
\hline L10 & Breweries & $10^{\circ} 28^{\prime} 40.07^{\prime \prime N}, 7^{\circ} 24^{\prime} 7.42^{\prime \prime E}$ \\
\hline L11 & Ungwa Mu’Azu & $10^{\circ} 29^{\prime} 17.15^{\prime \prime} \mathrm{N}, 7^{\circ} 22^{\prime} 56.89^{\prime \prime} \mathrm{E}$ \\
\hline L12 & Rigasa & $10^{\circ} 29^{\prime} 42.63^{\prime \prime} \mathrm{N}, 7^{\circ} 22^{\prime} 45.92^{\prime \prime} \mathrm{E}$ \\
\hline L13 & Maigiginya & $10^{\circ} 29^{\prime} 30.84 " \mathrm{~N}, 7^{\circ} 20^{\prime} 48.66^{\prime \prime} \mathrm{E}$ \\
\hline L14 & Romi & $10^{\circ} 29^{\prime} 10.65^{\prime \prime} \mathrm{N}, 7^{\circ} 20^{\prime} 31.50^{\prime \prime} \mathrm{E}$ \\
\hline L15 & Railway Bridge & $10^{\circ} 29^{\prime} 31.67 " \mathrm{~N}, 7^{\circ} 20^{\prime} 13.77^{\prime \prime} \mathrm{E}$ \\
\hline
\end{tabular}

Sampling Procedure and Laboratory Analysis: The sampling was done monthly for a period of one year between June 2016 and May 2017 thus, covering two metrological seasons. This sampling frequency and duration is in line with Adebayo (2014) and Esengul et al. (2014).

The grab sampling technique was employed in each sampling location. This was done by dipping high density polyethylene (HDPE) plastic bottles below the water surface at the center of the stream and ensuring that the mouth of the bottle faces the water current. Prior to sampling, the sample bottles were disinfected with methylated spirit and then thoroughly rinsed with the sample water before sample collection as recommended by APHA, (2005). The collected samples were stored in a cooler containing ice and delivered on the same day to the laboratory where they were refrigerated until analysis. However, DO, pH, TDS and EC were determined in-situ.

A portable dissolved oxygen meter (DO STARTER300D $\pm 1 \%$ made by OHAUS Corporation, USA) was used for the determination of dissolved oxygen while a Pocket-sized $\mathrm{pH}$ meter $\left(\mathrm{pHep}^{\circledR}, \pm 0.1\right.$ made by HANNA LTD, England) was used in determining $\mathrm{pH}$. TDS and EC were determined via a pocket-sized dissolved solids and conductivity meter with temperature compensation (TDS \& EC hold, $\pm 2 \%$ made by Griffin Company, USA). However, turbidity, total nitrogen and total phosphorus were analyzed by HACH $2100 \mathrm{~N}$ turbidimeter (made by HANNA, LTD, England), Kjeldahl auto distillation machine (Kjeltec $8200^{\mathrm{TM}}$ made by FOSS, Sweden) and Phosphorous meter 
(Colorimeter 257 made by Sherwood, USA) respectively. Determination of chloride ion $\left(\mathrm{Cl}^{-}\right)$ was achieved through Mohr's titrimetric method by using silver nitrate as titrant while heavy metals (Fe and $\mathrm{Mn}$ ) were analyzed through atomic absorption spectrophotometer (280FS AA made by Agilent Technology, USA). Glassware (BOD bottles, conical flasks, measuring cylinders, pipettes and burets) made by Kimax Company, England were used for titration during the determination of $\mathrm{Cl}^{-}, \mathrm{BOD}$ and COD. In addition, a handheld Global Position System navigator (Etrex 20x) made by Garmin, USA was used in determining the geographical locations of the sampled points.

Development of Water Quality Index: The Water Quality Index (WQI) developed was based on the Canadian Council of Ministers of Environment (CCME), which has been adopted by the Global Environmental Monitoring Systems (GEMS, 2007). The index is based on a combination of three factors:

Scope, $\mathbf{F}_{\mathbf{1}}$ - the number of variables whose objectives are not met

$F_{1}=\frac{\text { Number of failed variables }}{\text { Total number of variables }} \times 100$

Frequency, $\mathbf{F}_{2}$, - the frequency with which the objectives are not met.

$F_{2}=\frac{\text { Number of failed tests }}{\text { Total number of tests }} \times 100$

Amplitude, $\mathbf{F}_{3}$, - the amount by which the objectives are not met. $\mathrm{F}_{3}$ was calculated in three steps:

a) The number of times by which an individual concentration was greater than (or less than, when the objective is a minimum) the objective was termed an "excursion" and was estimated as follows;

b) $\quad$ excursion $_{i}=\frac{{\text { Failed } \text { Test }_{\text {Value }}}_{i}}{\text { objective }_{j}}-1$

For cases in which the test value must not exceed the objective:

excursion $_{i}=\frac{\text { Objective }_{j}}{\text { Failed Test value }_{i}}-1$

c) The collective amount by which individual tests were out of compliance was calculated by summing the excursions of individual tests from their objectives and dividing by the total number of tests (both those meeting objectives and those not meeting objectives). This variable, referred to as the normalized sum of excursions (nse), was calculated as:

nse $=\frac{\sum_{i=1}^{n} \text { excursion }_{i}}{\text { number of tests }}$

d) $\quad \mathrm{F}_{3}$ was thereafter calculated by an asymptotic function that scales the normalized sum of the excursions from objectives (nse) to yield a range between 0 and 100 as given in Equation (6)

$F_{3}=\frac{n s e}{0.01 n s e+0.01}$

The Canadian Council of Ministers of Environment Water Quality Index (CCME WQI) was then developed by substituting the values of $F_{1}, F_{2}$ and $\mathrm{F}_{3}$ into the Equation (7) given by CCME, 2001.

$W Q I=100-\frac{\sqrt{\left({F_{1}}^{2}+{F_{2}}^{2}+F_{3}{ }^{2}\right)}}{1.732}$

Equation (7) was employed in all the sampling locations and their respective results were computed. Thereafter, the results obtained were ranked into five categories as recommended by the Canadian Council of Ministers of Environment (CCME, 2001). These five categories for the assessment and protection of aquatic environment are as follows;

Excellent: (CCME WQI Value 95-100) - Water quality is protected with a virtual absence of threat or impairment; conditions very close to natural or pristine levels.

Good: (CCME WQI Value 80-94) - Water quality is protected with only a minor degree of threat or impairment; conditions rarely depart from natural or desirable levels.

Fair: (CCME WQI Value 65-79) - Water quality is usually protected but occasionally threatened or impaired; conditions sometimes depart from natural or desirable levels.

Marginal: (CCME WQI Value 45-64) - Water quality is frequently threatened or impaired; conditions often depart from natural or desirable levels.

Poor: (CCME WQI Value 0-44) - Water quality is almost always threatened or impaired; conditions usually depart from natural or desirable levels. 
Mapping of Water Quality Index: Inverse Distance Weighted Interpolation (IDW) method of the spatial analyst extension (ESRI, 2015) in the ArcGIS 10.5 was used in mapping the WQI within the catchment area. This is because Inverse Distance Weighted interpolation (IDW) assumes that the nearer a sample point is to the cell whose value is to be estimated, the more closely the cell's value will resemble the sample point's value. In other words, the principle underlying IDW is the Waldo Tobler's first law of Geography which states that "everything is related to everything else, but near things are more related than distant things".

IDW uses linear combination of weights at known points to estimate unknown location values (ESRI, 2015). That is, values at unknown locations $\hat{Z}\left(S_{0}\right)$ were determined by the weighting value $\lambda_{i}\left(S_{0}\right)$ and values at known locations $Z\left(S_{i}\right)$ expressed mathematically as shown in Equation (8), ESRI (2015).
$\hat{Z}\left(S_{0}\right)=\sum_{i=1}^{n} \lambda_{i}\left(S_{0}\right) \cdot Z\left(S_{i}\right)$

However, the weights $\lambda_{i}\left(S_{0}\right)$ were estimated through inverse distance from all points to the new points by applying equation (9), ESRI (2015).

$\lambda_{i}\left(S_{0}\right)=\frac{\frac{1}{\beta d\left(S_{0}, S_{1}\right)}}{\sum_{i=0}^{n} \overline{\beta d\left(S_{0}, S_{1}\right)}} ; \beta>1$

Where:

$\lambda_{i}=$ Weight for neighbor $i$ (the sum of weights must be unity to ensure an unbiased interpolator).

$\boldsymbol{d}\left(\boldsymbol{S}_{\mathbf{0}}, \boldsymbol{S}_{1}\right)=$ Distance from the new point to a known sample point.

$\boldsymbol{\beta}=$ Coefficient used to adjust the weights.

$\boldsymbol{n}=$ Total number of points in the neighbourhood analysis.

\section{RESULTS AND DISCUSSION}

The in-situ and laboratory results of the concerned water quality parameters in all the sampling sites (i.e sampling location L1 to L15) are shown in Table 2 to Table 13.

Table 2: Monthly variation of turbidity (NTU)

\begin{tabular}{|c|c|c|c|c|c|c|c|c|c|c|c|c|c|c|c|}
\hline & L1 & L2 & L3 & L4 & L5 & L6 & L7 & L8 & L9 & L10 & L11 & L12 & L13 & L14 & L15 \\
\hline Jun & 78.4 & 49.4 & 150.9 & 83.4 & 81.0 & 95.4 & 110.1 & 188.2 & 109.7 & 167.5 & 107.6 & 111.5 & 75.9 & 84.9 & 55.3 \\
\hline Jul & 92.0 & 58.0 & 176.5 & 98.1 & 95.1 & 111.8 & 128.2 & 221.3 & 128.5 & 196.4 & 126.1 & 130.5 & 87.9 & 99.9 & 65.1 \\
\hline Aug & 74.3 & 46.2 & 142.4 & 77.8 & 76.0 & 90.3 & 103.9 & 176.6 & 103.8 & 158.9 & 101.2 & 106.3 & 70.8 & 80.7 & 52.1 \\
\hline Sep & 41.4 & 27.8 & 79.9 & 43.3 & 42.6 & 50.4 & 57.7 & 100.4 & 58.2 & 88.9 & 56.8 & 59.1 & 39.3 & 44.6 & 28.9 \\
\hline Oct & 29.4 & 18.5 & 56.2 & 31.3 & 30.1 & 35.7 & 41.5 & 71.8 & 41.2 & 63.5 & 41.0 & 42.1 & 28.7 & 31.9 & 20.8 \\
\hline Nov & 26.8 & 8.7 & 4.3 & 9.1 & 9.4 & 6.9 & 4.9 & 1.8 & 5.2 & 2.3 & 5.5 & 4.6 & 17.1 & 9.1 & 21.2 \\
\hline Dec & 22.8 & 7.5 & 3.7 & 7.8 & 8.0 & 5.9 & 4.2 & 1.6 & 4.4 & 1.9 & 4.6 & 3.9 & 14.6 & 7.7 & 18.1 \\
\hline Jan & 21.4 & 7.0 & 3.4 & 7.3 & 7.7 & 5.5 & 3.9 & 1.5 & 4.1 & 1.8 & 4.4 & 3.7 & 13.8 & 7.3 & 17.1 \\
\hline Feb & 18.2 & 5.9 & 2.9 & 6.2 & 6.4 & 4.6 & 3.3 & 1.2 & 3.5 & 1.6 & 3.7 & 3.1 & 116 & 6.1 & 14.4 \\
\hline Mar & 12.2 & 3.9 & 1.9 & 4.2 & 4.3 & 3.1 & 2.2 & 0.9 & 2.3 & 1.0 & 2.4 & 2.1 & 7.7 & 4.1 & 9.6 \\
\hline Apr & 8.7 & 2.8 & 1.4 & 2.9 & 3.0 & 2.2 & 1.6 & 0.6 & 1.7 & 0.7 & 1.7 & 1.5 & 5.5 & 2.9 & 6.8 \\
\hline May & 62.1 & 38.9 & 119.5 & 66.1 & 63.8 & 75.3 & 87.2 & 149.9 & 86.9 & 132.9 & 85.9 & 88.2 & 60.2 & 67.1 & 43.8 \\
\hline
\end{tabular}

NTU = Naphelometric Turbidity Unit. L1, L2, L3,......L15 = Sampling Locations $1,2,3, \ldots \ldots \ldots, 15$ in that order.

Table 3: Monthly variation of total dissolved solids ( $\mathrm{mg} / \mathrm{L}$ )

\begin{tabular}{cccccccccccccccc}
\hline & L1 & L2 & L3 & L4 & L5 & L6 & L7 & L8 & L9 & L10 & L11 & L12 & L13 & L14 & L15 \\
\hline Jun & 81 & 88 & 84 & 83 & 80 & 401 & 93 & 460 & 82 & 525 & 95 & 498 & 80 & 94 & 80 \\
Jul & 76 & 85 & 79 & 80 & 75 & 387 & 82 & 408 & 80 & 504 & 88 & 476 & 75 & 89 & 77 \\
Aug & 65 & 80 & 62 & 73 & 66 & 345 & 77 & 388 & 69 & 474 & 83 & 454 & 69 & 85 & 71 \\
Sep & 60 & 76 & 61 & 70 & 58 & 333 & 68 & 351 & 66 & 470 & 78 & 422 & 67 & 79 & 69 \\
Oct & 61 & 75 & 59 & 65 & 59 & 329 & 75 & 392 & 71 & 485 & 81 & 451 & 77 & 84 & 77 \\
Nov & 93 & 121 & 94 & 100 & 95 & 389 & 98 & 466 & 91 & 505 & 97 & 468 & 93 & 114 & 94 \\
Dec & 105 & 127 & 103 & 113 & 103 & 542 & 103 & 561 & 102 & 562 & 105 & 529 & 104 & 127 & 103 \\
Jan & 106 & 123 & 107 & 116 & 107 & 576 & 105 & 598 & 107 & 604 & 107 & 532 & 108 & 132 & 108 \\
Feb & 97 & 115 & 97 & 106 & 99 & 550 & 99 & 569 & 97 & 609 & 99 & 524 & 98 & 124 & 99 \\
Mar & 100 & 116 & 102 & 108 & 101 & 518 & 100 & 587 & 101 & 600 & 101 & 508 & 102 & 121 & 101 \\
Apr & 90 & 110 & 88 & 99 & 93 & 507 & 91 & 529 & 91 & 593 & 89 & 504 & 92 & 116 & 92 \\
May & 79 & 86 & 78 & 86 & 82 & 502 & 91 & 506 & 82 & 547 & 87 & 502 & 81 & 102 & 81 \\
\hline
\end{tabular}

L1, L2, L3, L15 = Sampling Locations $1,2,3, \ldots \ldots \ldots, 15$ in that order. 
Table 4: Monthly variation of $\mathrm{pH}$

\begin{tabular}{|c|c|c|c|c|c|c|c|c|c|c|c|c|c|c|c|}
\hline & L1 & L2 & L3 & L4 & L5 & L6 & L7 & L8 & L9 & L10 & L11 & L12 & L13 & L14 & L15 \\
\hline Jun & 7.2 & 7.3 & 7.3 & 6.7 & 7.3 & 6.3 & 7.2 & 6.4 & 7.2 & 8.9 & 7.2 & 6.5 & 7.2 & 7.4 & 7.2 \\
\hline Jul & 6.8 & 7.3 & 6.9 & 6.7 & 6.8 & 6.3 & 6.8 & 6.3 & 6.9 & 8.8 & 6.8 & 6.4 & 6.7 & 7.3 & 6.8 \\
\hline Aug & 6.8 & 6.8 & 6.8 & 6.5 & 6.7 & 6.2 & 6.7 & 6.2 & 6.8 & 8.7 & 6.7 & 6.4 & 6.7 & 7.3 & 6.7 \\
\hline Sep & 6.9 & 6.7 & 6.8 & 6.6 & 6.8 & 6.4 & 6.8 & 6.3 & 6.9 & 8.9 & 6.9 & 6.3 & 6.8 & 7.4 & 6.8 \\
\hline Oct & 7.2 & 6.8 & 7.3 & 6.8 & 7.4 & 6.6 & 7.3 & 6.5 & 6.7 & 8.9 & 7.2 & 6.4 & 7.2 & 7.5 & 7.3 \\
\hline Nov & 7.4 & 6.8 & 7.5 & 6.7 & 7.6 & 6.8 & 7.7 & 6.6 & 6.9 & 9.0 & 7.4 & 6.7 & 7.4 & 7.6 & 7.5 \\
\hline Dec & 7.4 & 7.4 & 7.4 & 6.8 & 7.4 & 6.8 & 7.5 & 6.8 & 7.2 & 9.1 & 7.5 & 6.8 & 7.6 & 7.5 & 7.6 \\
\hline Jan & 7.5 & 7.4 & 7.5 & 7.3 & 7.5 & 6.9 & 7.4 & 7.1 & 7.4 & 9.2 & 7.7 & 7.9 & 7.8 & 7.7 & 7.7 \\
\hline Feb & 7.6 & 7.5 & 7.5 & 7.3 & 7.3 & 6.8 & 7.5 & 7.3 & 7.4 & 9.1 & 7.8 & 7.2 & 7.7 & 7.7 & 7.7 \\
\hline Mar & 7.3 & 7.6 & 7.4 & 7.4 & 7.4 & 6.7 & 7.4 & 7.4 & 7.4 & 9.3 & 7.9 & 7.4 & 7.8 & 7.8 & 7.8 \\
\hline Apr & 7.5 & 7.3 & 7.4 & 7.2 & 7.5 & 6.6 & 7.6 & 6.8 & 7.3 & 9.2 & 7.5 & 6.8 & 7.3 & 7.6 & 7.4 \\
\hline May & 7.2 & 7.3 & 7.3 & 6.9 & 7.4 & 6.4 & 7.3 & 6.3 & 7.2 & 9.0 & 7.3 & 6.4 & 7.1 & 7.5 & 7.2 \\
\hline
\end{tabular}

$\mathrm{L} 1, \mathrm{~L} 2, \mathrm{~L} 3, \ldots \ldots ., \mathrm{L} 15$ = Sampling Locations $1,2,3, \ldots \ldots \ldots, 15$ in that order.

Table 5: Monthly variation of chloride ion $(\mathrm{mg} / \mathrm{L})$

\begin{tabular}{|c|c|c|c|c|c|c|c|c|c|c|c|c|c|c|c|}
\hline & L1 & L2 & L3 & L4 & L5 & L6 & L7 & L8 & L9 & L10 & L11 & L12 & L13 & L14 & L15 \\
\hline Jun & 13.28 & 16.65 & 14.78 & 14.35 & 12.88 & 194.52 & 19.64 & 227.64 & 13.02 & 265.91 & 21.14 & 249.77 & 12.69 & 20.52 & 12.53 \\
\hline Aug & 3.91 & 12.14 & 2.42 & 8.69 & 4.66 & 162.80 & 10.66 & 187.03 & 6.16 & 236.59 & 14.40 & 225.05 & 6.54 & 15.66 & 7.28 \\
\hline Sep & 0.92 & 10.26 & 2.04 & 7.18 & 0.17 & 156.46 & 5.79 & 165.98 & 4.66 & 234.71 & 11.40 & 207.07 & 5.41 & 12.66 & 6.16 \\
\hline Nov & 19.64 & 35.45 & 20.39 & 24.16 & 20.77 & 187.80 & 22.64 & 230.64 & 18.52 & 254.63 & 21.89 & 232.92 & 19.64 & 32.13 & 20.39 \\
\hline Dec & 26.39 & 38.45 & 25.26 & 31.33 & 25.64 & 273.25 & 25.64 & 284.40 & 24.89 & 286.59 & 26.39 & 267.01 & 26.01 & 39.25 & 25.64 \\
\hline Jan & 27.13 & 36.58 & 27.88 & 32.84 & 27.88 & 292.28 & 26.76 & 305.08 & 27.51 & 310.27 & 27.51 & 268.87 & 28.26 & 41.87 & 28.26 \\
\hline Apr & 18.15 & 29.06 & 17.02 & 23.40 & 19.64 & 253.47 & 18.90 & 266.36 & 18.90 & 303.88 & 17.77 & 252.77 & 19.27 & 33.26 & 19.27 \\
\hline May & 12.15 & 15.53 & 11.40 & 15.86 & 12.53 & 250.86 & 18.52 & 253.20 & 13.65 & 278.32 & 16.27 & 251.64 & 12.53 & 25.02 & 13.28 \\
\hline
\end{tabular}

L1, L2, L3, .....,L15 = Sampling Locations $1,2,3, \ldots \ldots \ldots, 15$ in that order.

Table 6: Monthly variation of electrical conductivity $(\mu \mathrm{S} / \mathrm{cm})$

\begin{tabular}{|c|c|c|c|c|c|c|c|c|c|c|c|c|c|c|c|}
\hline & L1 & L2 & $\mathbf{L 3}$ & L4 & L5 & L6 & L7 & L8 & L9 & L10 & L11 & L12 & L13 & L14 & L15 \\
\hline Jun & 122 & 131 & 126 & 124 & 120 & 601 & 136 & 690 & 123 & 789 & 143 & 747 & 120 & 140 & 120 \\
\hline Jul & 114 & 127 & 119 & 120 & 113 & 580 & 139 & 611 & 120 & 757 & 132 & 713 & 113 & 133 & 115 \\
\hline Aug & 97 & 119 & 93 & 109 & 99 & 516 & 123 & 582 & 103 & 711 & 125 & 681 & 104 & 127 & 106 \\
\hline Sep & 89 & 114 & 92 & 105 & 87 & 499 & 115 & 526 & 99 & 706 & 117 & 633 & 101 & 119 & 103 \\
\hline Oct & 91 & 112 & 88 & 97 & 89 & 493 & 102 & 587 & 106 & 728 & 121 & 676 & 116 & 126 & 116 \\
\hline Nov & 139 & 181 & 141 & 150 & 142 & 583 & 113 & 698 & 136 & 759 & 145 & 702 & 139 & 171 & 141 \\
\hline Dec & 157 & 189 & 154 & 169 & 155 & 812 & 155 & 841 & 153 & 844 & 157 & 793 & 156 & 190 & 155 \\
\hline Jan & 159 & 184 & 161 & 173 & 161 & 863 & 158 & 896 & 160 & 907 & 160 & 798 & 162 & 197 & 162 \\
\hline Feb & 146 & 171 & 145 & 158 & 148 & 823 & 149 & 853 & 145 & 915 & 148 & 786 & 147 & 186 & 148 \\
\hline Mar & 150 & 173 & 153 & 162 & 152 & 776 & 150 & 880 & 151 & 901 & 152 & 762 & 153 & 181 & 151 \\
\hline Apr & 135 & 164 & 132 & 148 & 139 & 759 & 137 & 793 & 137 & 890 & 134 & 755 & 138 & 174 & 138 \\
\hline May & 119 & 128 & 117 & 128 & 120 & 752 & 136 & 758 & 123 & 822 & 130 & 752 & 120 & 152 & 122 \\
\hline
\end{tabular}

$\mu \mathrm{S} / \mathrm{cm}=$ Micro Mohs per centimeter. L1, L2, L3, ........L15 = Sampling Locations $1,2,3, \ldots \ldots \ldots, 15$ in that order.

Table 7: Monthly variation of dissolved oxygen $(\mathrm{mg} / \mathrm{L})$

\begin{tabular}{|c|c|c|c|c|c|c|c|c|c|c|c|c|c|c|c|}
\hline & L1 & L2 & L3 & L4 & L5 & L6 & L7 & L8 & L9 & L10 & L11 & L12 & L13 & L14 & L15 \\
\hline Jun & 7.80 & 6.85 & 7.72 & 6.51 & 7.27 & 4.72 & 7.23 & 4.80 & 7.09 & 4.24 & 7.01 & 4.09 & 7.58 & 6.88 & 7.58 \\
\hline Jul & 8.18 & 6.92 & 8.15 & 6.68 & 7.32 & 4.77 & 7.19 & 4.86 & 7.13 & 4.37 & 7.04 & 4.83 & 8.02 & 6.97 & 8.01 \\
\hline Aug & 8.22 & 6.88 & 8.23 & 6.23 & 6.84 & 4.56 & 6.77 & 4.73 & 6.81 & 4.29 & 6.75 & 4.52 & 7.79 & 6.56 & 7.76 \\
\hline Sep & 8.10 & 6.32 & 8.06 & 5.97 & 6.50 & 4.22 & 6.59 & 4.39 & 6.54 & 4.06 & 6.42 & 4.49 & 7.08 & 6.39 & 7.08 \\
\hline Oct & 7.96 & 6.11 & 7.85 & 5.46 & 6.17 & 3.97 & 6.14 & 4.12 & 6.10 & 3.71 & 5.97 & 4.17 & 6.48 & 6.11 & 6.44 \\
\hline Nov & 7.48 & 5.73 & 7.52 & 5.17 & 5.88 & 3.66 & 5.09 & 3.90 & 5.07 & 3.55 & 4.81 & 3.91 & 5.02 & 5.60 & 5.04 \\
\hline Dec & 7.25 & 5.28 & 6.99 & 4.86 & 5.69 & 3.39 & 4.51 & 3.44 & 4.10 & 3.46 & 3.77 & 3.91 & 4.36 & 4.81 & 4.38 \\
\hline Jan & 6.11 & 4.86 & 6.27 & 4.39 & 4.87 & 3.28 & 4.12 & 3.37 & 4.02 & 2.61 & 3.64 & 3.86 & 4.05 & 4.48 & 4.09 \\
\hline Feb & 5.87 & 4.77 & 5.93 & 4.33 & 4.61 & 3.23 & 3.95 & 3.15 & 3.56 & 2.19 & 3.49 & 3.54 & 3.98 & 4.11 & 4.01 \\
\hline Mar & 5.25 & 4.50 & 5.37 & 4.20 & 4.48 & 3.15 & 3.86 & 3.11 & 3.44 & 2.43 & 3.16 & 3.29 & 3.82 & 3.93 & 3.82 \\
\hline Apr & 5.18 & 4.19 & 5.06 & 4.05 & 4.22 & 3.11 & 3.67 & 2.18 & 3.23 & 2.29 & 3.07 & 3.08 & 3.64 & 3.62 & 3.64 \\
\hline May & 6.39 & 5.04 & 6.42 & 5.72 & 5.96 & 3.93 & 5.38 & 2.97 & 5.84 & 3.14 & 5.33 & 3.61 & 5.20 & 5.09 & 5.19 \\
\hline
\end{tabular}

$\mathrm{L} 1, \mathrm{~L} 2, \mathrm{~L} 3, \ldots \ldots . \mathrm{L} 15=$ Sampling Locations $1,2,3, \ldots \ldots \ldots, 15$ in that order. 
Table 8: Monthly variation of 5-days Biochemical Oxygen Demand (mg/L)

\begin{tabular}{|c|c|c|c|c|c|c|c|c|c|c|c|c|c|c|c|}
\hline & L1 & L2 & $\mathbf{L 3}$ & L4 & L5 & L6 & L7 & L8 & L9 & L10 & L11 & L12 & L13 & L14 & L15 \\
\hline Jun & 0.43 & 0.55 & 1.63 & 1.01 & 1.20 & 1.81 & 1.85 & 2.04 & 1.70 & 2.16 & 1.72 & 1.67 & 0.41 & 0.95 & 0.17 \\
\hline Jul & 0.48 & 0.58 & 1.37 & 1.04 & 1.23 & 1.84 & 1.81 & 2.09 & 1.75 & 2.24 & 1.59 & 1.96 & 0.43 & 0.99 & 0.18 \\
\hline Aug & 0.50 & 0.59 & 1.33 & 1.06 & 1.30 & 1.86 & 1.73 & 2.05 & 1.73 & 2.32 & 1.46 & 2.04 & 0.43 & 1.08 & 0.18 \\
\hline Sep & 0.59 & 0.64 & 1.42 & 1.17 & 1.52 & 2.02 & 1.72 & 2.12 & 1.87 & 2.41 & 1.43 & 2.10 & 0.47 & 1.16 & 0.19 \\
\hline Oct & 0.61 & 0.69 & 1.68 & 1.24 & 1.57 & 2.07 & 1.77 & 2.19 & 1.85 & 2.46 & 1.49 & 2.19 & 0.54 & 1.35 & 0.22 \\
\hline Nov & 0.65 & 0.74 & 1.84 & 1.31 & 1.65 & 2.12 & 1.94 & 2.55 & 1.96 & 2.66 & 1.54 & 2.36 & 0.69 & 1.47 & 0.28 \\
\hline Dec & 0.66 & 0.77 & 1.94 & 1.44 & 1.74 & 2.21 & 2.08 & 2.68 & 1.99 & 2.88 & 1.97 & 2.42 & 0.76 & 1.54 & 0.31 \\
\hline Jan & 0.67 & 0.83 & 1.98 & 1.50 & 1.83 & 2.34 & 2.23 & 2.72 & 2.41 & 2.02 & 2.18 & 2.49 & 0.83 & 1.58 & 0.34 \\
\hline Feb & 0.66 & 0.84 & 2.00 & 1.61 & 1.96 & 2.51 & 2.40 & 2.71 & 2.57 & 2.07 & 2.25 & 2.63 & 0.86 & 1.68 & 0.35 \\
\hline Mar & 0.63 & 0.83 & 1.93 & 1.57 & 1.94 & 2.46 & 2.44 & 2.69 & 2.55 & 1.99 & 2.40 & 2.60 & 0.81 & 1.66 & 0.33 \\
\hline Apr & 0.52 & 0.61 & 1.91 & 1.38 & 1.59 & 2.51 & 1.98 & 1.76 & 2.93 & 1.31 & 2.58 & 2.66 & 0.56 & 1.23 & 0.23 \\
\hline May & 0.42 & 0.51 & 1.88 & 0.97 & 1.13 & 2.03 & 1.91 & 1.38 & 1.79 & 2.02 & 1.86 & 1.86 & 0.39 & 0.87 & 0.16 \\
\hline
\end{tabular}

L1 $, \mathrm{L} 2, \mathrm{~L} 3, \ldots \ldots . . \mathrm{L} 15$ = Sampling Locations $1,2,3, \ldots \ldots \ldots, 15$ in that order.

Table 9: Monthly variation of Chemical Oxygen Demand (mg/L)

\begin{tabular}{|c|c|c|c|c|c|c|c|c|c|c|c|c|c|c|c|}
\hline & L1 & L2 & L3 & L4 & L5 & L6 & L7 & L8 & L9 & L10 & L11 & L12 & L13 & L14 & L15 \\
\hline Jun & 35.08 & 36.49 & 49.92 & 40.84 & 41.94 & 46.50 & 53.25 & 61.72 & 52.35 & 58.23 & 48.33 & 53.10 & 37.91 & 43.00 & 35.91 \\
\hline Jul & 33.79 & 35.00 & 47.88 & 39.18 & 40.23 & 44.60 & 51.08 & 58.17 & 50.18 & 55.82 & 46.06 & 50.61 & 36.13 & 39.96 & 33.37 \\
\hline Aug & 34.32 & 35.55 & 48.64 & 39.79 & 40.86 & 45.30 & 51.88 & 59.08 & 50.97 & 56.70 & 46.79 & 51.41 & 36.70 & 40.59 & 33.90 \\
\hline Sep & 36.04 & 37.33 & 51.07 & 41.78 & 42.91 & 47.57 & 54.48 & 62.05 & 53.53 & 59.55 & 49.13 & 53.98 & 38.54 & 42.62 & 35.59 \\
\hline Oct & 37.99 & 39.35 & 53.83 & 44.04 & 45.22 & 50.14 & 57.42 & 65.40 & 56.41 & 62.76 & 51.78 & 56.89 & 40.62 & 44.92 & 37.51 \\
\hline Nov & 40.12 & 41.55 & 56.84 & 46.51 & 47.76 & 52.95 & 60.64 & 69.06 & 59.58 & 66.28 & 54.69 & 60.09 & 42.90 & 47.45 & 39.63 \\
\hline Dec & 41.46 & 42.95 & 58.76 & 48.07 & 49.36 & 54.73 & 62.68 & 71.38 & 61.58 & 68.50 & 56.53 & 62.11 & 44.34 & 49.04 & 40.96 \\
\hline Jan & 43.26 & 44.80 & 61.29 & 50.15 & 51.50 & 57.10 & 65.39 & 74.48 & 64.25 & 71.47 & 58.97 & 64.79 & 46.26 & 51.16 & 42.73 \\
\hline Feb & 43.84 & 45.41 & 62.13 & 50.83 & 51.18 & 57.93 & 66.34 & 75.56 & 65.18 & 72.51 & 59.83 & 65.74 & 46.93 & 51.91 & 43.35 \\
\hline Mar & 44.39 & 45.98 & 62.91 & 51.47 & 52.85 & 58.59 & 67.09 & 76.42 & 65.93 & 73.34 & 60.51 & 66.48 & 47.46 & 52.49 & 43.84 \\
\hline Apr & 45.07 & 46.68 & 63.86 & 52.25 & 53.66 & 59.49 & 68.13 & 77.59 & 66.94 & 74.46 & 61.44 & 67.50 & 48.19 & 53.30 & 44.51 \\
\hline May & 36.21 & 37.51 & 51.32 & 41.98 & 43.11 & 47.80 & 54.74 & 62.35 & 53.78 & 59.82 & 49.37 & 54.24 & 38.72 & 42.83 & 35.77 \\
\hline
\end{tabular}

L1, L2, L3, .......L15 = Sampling Locations $1,2,3, \ldots \ldots \ldots, 15$ in that order.

Table 10: Monthly variation of total nitrogen $(\mathrm{mg} / \mathrm{L})$

\begin{tabular}{|c|c|c|c|c|c|c|c|c|c|c|c|c|c|c|c|}
\hline & L1 & L2 & $\mathbf{L 3}$ & L4 & L5 & L6 & L7 & L8 & L9 & L10 & L11 & L12 & L13 & L14 & L15 \\
\hline Jun & 0.96 & 0.79 & 0.73 & 0.80 & 0.80 & 0.67 & 0.73 & 0.87 & 0.74 & 0.82 & 0.74 & 0.74 & 0.87 & 0.79 & 0.91 \\
\hline Jul & 0.88 & 0.73 & 0.67 & 0.73 & 0.74 & 0.63 & 0.68 & 0.82 & 0.69 & 0.77 & 0.69 & 0.70 & 0.80 & 0.73 & 0.84 \\
\hline Aug & 0.85 & 0.70 & 0.65 & 0.71 & 0.72 & 0.59 & 0.65 & 0.77 & 0.66 & 0.72 & 0.67 & 0.66 & 0.78 & 0.71 & 0.81 \\
\hline Sep & 0.83 & 0.69 & 0.64 & 0.69 & 0.69 & 0.64 & 0.64 & 0.83 & 0.65 & 0.78 & 0.65 & 0.71 & 0.76 & 0.69 & 0.79 \\
\hline Oct & 0.90 & 0.75 & 0.69 & 0.75 & 0.76 & 0.73 & 0.70 & 0.95 & 0.70 & 0.89 & 0.71 & 0.81 & 0.81 & 0.75 & 0.85 \\
\hline Nov & 1.11 & 0.92 & 0.85 & 0.92 & 0.93 & 0.92 & 0.85 & 1.20 & 0.85 & 1.12 & 0.86 & 1.02 & 1.00 & 0.92 & 1.05 \\
\hline Dec & 1.24 & 1.03 & 0.95 & 1.04 & 1.05 & 1.22 & 0.96 & 1.59 & 0.96 & 1.49 & 0.98 & 1.36 & 1.13 & 1.04 & 1.18 \\
\hline Jan & 1.39 & 1.15 & 1.06 & 1.16 & 1.16 & 1.27 & 1.07 & 1.66 & 1.07 & 1.56 & 1.08 & 1.42 & 1.26 & 1.15 & 1.32 \\
\hline Feb & 1.77 & 1.47 & 1.36 & 1.48 & 1.48 & 1.41 & 1.36 & 1.84 & 1.37 & 1.72 & 1.38 & 1.57 & 1.61 & 1.47 & 1.68 \\
\hline Mar & 1.93 & 1.60 & 1.48 & 1.61 & 1.62 & 1.46 & 1.49 & 1.91 & 1.50 & 1.79 & 1.51 & 1.63 & 1.75 & 1.61 & 1.83 \\
\hline Apr & 2.18 & 1.80 & 1.66 & 1.81 & 1.82 & 1.55 & 1.67 & 2.02 & 1.68 & 1.89 & 1.70 & 1.72 & 1.98 & 1.81 & 2.07 \\
\hline May & 1.43 & 1.18 & 1.09 & 1.19 & 1.19 & 1.02 & 1.09 & 1.33 & 1.10 & 1.25 & 1.11 & 1.13 & 1.30 & 1.18 & 1.36 \\
\hline
\end{tabular}

L1, L2, L3,$\ldots \ldots . ., L 15$ = Sampling Locations $1,2,3, \ldots \ldots \ldots, 15$ in that order.

Table 11: Monthly variation of total phosphorus $(\mathrm{mg} / \mathrm{L})$

\begin{tabular}{|c|c|c|c|c|c|c|c|c|c|c|c|c|c|c|c|}
\hline & L1 & L2 & L3 & L4 & L5 & L6 & L7 & L8 & L9 & L10 & L11 & L12 & L13 & L14 & L15 \\
\hline Jun & 0.140 & 0.115 & 0.107 & 0.117 & 0.117 & 0.098 & 0.107 & 0.114 & 0.108 & 0.120 & 0.109 & 0.108 & 0.127 & 0.115 & 0.133 \\
\hline Jul & 0.128 & 0.107 & 0.098 & 0.107 & 0.108 & 0.092 & 0.099 & 0.106 & 0.101 & 0.112 & 0.102 & 0.102 & 0.117 & 0.107 & 0.123 \\
\hline Aug & 0.124 & 0.102 & 0.095 & 0.104 & 0.105 & 0.101 & 0.095 & 0.119 & 0.096 & 0.105 & 0.096 & 0.096 & 0.114 & 0.104 & 0.118 \\
\hline Sep & 0.121 & 0.101 & 0.093 & 0.101 & 0.102 & 0.097 & 0.093 & 0.115 & 0.095 & 0.114 & 0.096 & 0.104 & 0.111 & 0.101 & 0.115 \\
\hline Oct & 0.131 & 0.110 & 0.100 & 0.110 & 0.111 & 0.107 & 0.102 & 0.122 & 0.103 & 0.130 & 0.104 & 0.118 & 0.118 & 0.110 & 0.124 \\
\hline Nov & 0.162 & 0.134 & 0.124 & 0.134 & 0.136 & 0.129 & 0.124 & 0.148 & 0.125 & 0.164 & 0.126 & 0.149 & 0.146 & 0.134 & 0.153 \\
\hline Dec & 0.181 & 0.150 & 0.139 & 0.152 & 0.153 & 0.145 & 0.140 & 0.193 & 0.141 & 0.218 & 0.141 & 0.200 & 0.165 & 0.152 & 0.172 \\
\hline Jan & 0.203 & 0.168 & 0.155 & 0.169 & 0.169 & 0.161 & 0.156 & 0.217 & 0.156 & 0.228 & 0.157 & 0.207 & 0.184 & 0.168 & 0.193 \\
\hline Feb & 0.258 & 0.215 & 0.200 & 0.216 & 0.216 & 0.204 & 0.199 & 0.236 & 0.200 & 0.251 & 0.201 & 0.229 & 0.235 & 0.215 & 0.245 \\
\hline Mar & 0.282 & 0.234 & 0.216 & 0.235 & 0.237 & 0.226 & 0.218 & 0.244 & 0.219 & 0.261 & 0.220 & 0.238 & 0.256 & 0.235 & 0.267 \\
\hline Apr & 0.318 & 0.263 & 0.242 & 0.264 & 0.266 & 0.251 & 0.244 & 0.258 & 0.245 & 0.276 & 0.246 & 0.251 & 0.289 & 0.264 & 0.302 \\
\hline May & 0.209 & 0.172 & 0.159 & 0.174 & 0.174 & 0.164 & 0.159 & 0.160 & 0.161 & 0.183 & 0.163 & 0.165 & 0.190 & 0.172 & 0.198 \\
\hline
\end{tabular}

L1, L2, L3, .....,L15 = Sampling Locations $1,2,3, \ldots \ldots \ldots, 15$ in that order. 
Table 12: Monthly variation of iron $(\mathrm{mg} / \mathrm{L})$

\begin{tabular}{|c|c|c|c|c|c|c|c|c|c|c|c|c|c|c|c|}
\hline & L1 & L2 & L3 & L4 & L5 & L6 & L7 & L8 & L9 & L10 & L11 & L12 & L13 & L14 & L15 \\
\hline Jun & 0.181 & 0.188 & 0.257 & 0.210 & 0.216 & 0.239 & 0.269 & 0.312 & 0.265 & 0.292 & 0.243 & 0.265 & 0.186 & 0.210 & 0.175 \\
\hline Jul & 0.165 & 0.172 & 0.235 & 0.193 & 0.198 & 0.220 & 0.248 & 0.287 & 0.243 & 0.269 & 0.222 & 0.245 & 0.169 & 0.194 & 0.159 \\
\hline Aug & 0.208 & 0.216 & 0.296 & 0.242 & 0.248 & 0.275 & 0.310 & 0.359 & 0.300 & 0.337 & 0.275 & 0.306 & 0.213 & 0.243 & 0.200 \\
\hline Sep & 0.236 & 0.245 & 0.298 & 0.274 & 0.281 & 0.312 & 0.351 & 0.407 & 0.345 & 0.382 & 0.316 & 0.346 & 0.242 & 0.275 & 0.228 \\
\hline Oct & 0.239 & 0.249 & 0.300 & 0.279 & 0.286 & 0.317 & 0.356 & 0.413 & 0.350 & 0.387 & 0.320 & 0.351 & 0.245 & 0.280 & 0.230 \\
\hline Nov & 0.241 & 0.251 & 0.343 & 0.281 & 0.288 & 0.319 & 0.359 & 0.416 & 0.353 & 0.390 & 0.323 & 0.354 & 0.247 & 0.282 & 0.232 \\
\hline Dec & 0.248 & 0.258 & 0.353 & 0.289 & 0.298 & 0.330 & 0.371 & 0.430 & 0.365 & 0.403 & 0.334 & 0.366 & 0.254 & 0.290 & 0.239 \\
\hline Jan & 0.294 & 0.306 & 0.419 & 0.342 & 0.351 & 0.389 & 0.437 & 0.507 & 0.430 & 0.475 & 0.394 & 0.431 & 0.302 & 0.343 & 0.284 \\
\hline Feb & 0.399 & 0.415 & 0.568 & 0.464 & 0.476 & 0.528 & 0.594 & 0.689 & 0.584 & 0.646 & 0.535 & 0.586 & 0.409 & 0.465 & 0.385 \\
\hline Mar & 0.463 & 0.482 & 0.659 & 0.539 & 0.553 & 0.613 & 0.689 & 0.799 & 0.662 & 0.749 & 0.606 & 0.680 & 0.475 & 0.540 & 0.447 \\
\hline Apr & 0.343 & 0.357 & 0.488 & 0.400 & 0.411 & 0.456 & 0.513 & 0.595 & 0.505 & 0.558 & 0.462 & 0.506 & 0.352 & 0.401 & 0.331 \\
\hline May & 0.249 & 0.259 & 0.291 & 0.290 & 0.298 & 0.311 & 0.350 & 0.406 & 0.344 & 0.381 & 0.314 & 0.345 & 0.255 & 0.291 & 0.240 \\
\hline
\end{tabular}

L1, L2, L3, ......L15 = Sampling Locations $1,2,3, \ldots \ldots \ldots, 15$ in that order.

Table 13: Monthly variation of manganese $(\mathrm{mg} / \mathrm{L})$

\begin{tabular}{cccccccccccccccc}
\multicolumn{10}{c}{ Table 13: Monthly variation of manganese $(\mathrm{mg} / \mathrm{L})$} \\
\hline & $\mathbf{L 1}$ & $\mathbf{L 2}$ & $\mathbf{L 3}$ & $\mathbf{L 4}$ & $\mathbf{L 5}$ & $\mathbf{L 6}$ & $\mathbf{L 7}$ & $\mathbf{L 8}$ & $\mathbf{L 9}$ & $\mathbf{L 1 0}$ & L11 & L12 & L13 & L14 & L15 \\
\hline Jun & 0.064 & ND & 0.070 & 0.074 & 0.076 & 0.085 & 0.095 & 0.099 & 0.092 & 0.103 & 0.085 & 0.093 & 0.066 & 0.075 & 0.062 \\
Jul & 0.106 & ND & 0.119 & 0.123 & 0.125 & 0.141 & 0.157 & 0.166 & 0.155 & 0.172 & 0.143 & 0.156 & 0.110 & 0.124 & 0.103 \\
Aug & 0.121 & ND & 0.136 & 0.140 & 0.143 & 0.161 & 0.179 & 0.190 & 0.176 & 0.196 & 0.163 & 0.177 & 0.126 & 0.141 & 0.119 \\
Sep & 0.143 & 0.011 & 0.155 & 0.166 & 0.170 & 0.190 & 0.212 & 0.225 & 0.209 & 0.233 & 0.193 & 0.211 & 0.150 & 0.167 & 0.141 \\
Oct & 0.168 & 0.019 & 0.186 & 0.195 & 0.214 & 0.247 & 0.275 & 0.291 & 0.271 & 0.301 & 0.250 & 0.272 & 0.176 & 0.200 & 0.166 \\
Nov & 0.192 & 0.025 & 0.200 & 0.254 & 0.260 & 0.292 & 0.326 & 0.345 & 0.321 & 0.357 & 0.296 & 0.323 & 0.197 & 0.256 & 0.185 \\
Dec & 0.246 & 0.028 & 0.255 & 0.285 & 0.291 & 0.326 & 0.364 & 0.386 & 0.359 & 0.399 & 0.331 & 0.361 & 0.257 & 0.286 & 0.242 \\
Jan & 0.285 & 0.037 & 0.312 & 0.331 & 0.338 & 0.380 & 0.424 & 0.449 & 0.417 & 0.464 & 0.385 & 0.419 & 0.298 & 0.333 & 0.280 \\
Feb & 0.310 & 0.043 & 0.346 & 0.359 & 0.366 & 0.412 & 0.459 & 0.486 & 0.451 & 0.502 & 0.417 & 0.454 & 0.323 & 0.362 & 0.304 \\
Mar & 0.364 & 0.056 & 0.374 & 0.422 & 0.428 & 0.482 & 0.537 & 0.569 & 0.529 & 0.588 & 0.488 & 0.532 & 0.377 & 0.423 & 0.355 \\
Apr & 0.249 & 0.031 & 0.322 & 0.289 & 0.294 & 0.330 & 0.368 & 0.390 & 0.362 & 0.403 & 0.334 & 0.364 & 0.259 & 0.290 & 0.244 \\
May & 0.139 & 0.018 & 0.200 & 0.161 & 0.165 & 0.186 & 0.207 & 0.219 & 0.203 & 0.226 & 0.188 & 0.198 & 0.145 & 0.163 & 0.136 \\
\hline
\end{tabular}

$\mathrm{ND}=$ Not Detected. L1, L2, L3, L15 = Sampling Locations $1,2,3, \ldots \ldots \ldots, 15$ in that order.

The data displayed in Table 3 to 14 were subjected into the Canadian Water Quality Index models across all the sampling locations and the values obtained are shown in Table 14.

Table 14: Summary Canadian WQI of Sampling locations

\begin{tabular}{llcc}
\hline Location Code & Location Name & WQI & Interpretation \\
\hline L1 & Malali & 56.31 & Marginal \\
L2 & Kwarau & 60.80 & Marginal \\
L3 & NNPC & 54.00 & Marginal \\
L4 & Kuyi & 52.83 & Marginal \\
L5 & Barnawa & 52.91 & Marginal \\
L6 & Kutimbi & 25.47 & Poor \\
L7 & Kigo & 46.22 & Marginal \\
L8 & Living Faith & 20.55 & Poor \\
L9 & Down Quarters & 44.95 & Marginal \\
L10 & Breweries & 17.77 & Poor \\
L11 & Ungwa Mu'azu & 46.58 & Marginal \\
L12 & Rigasa & 24.11 & Poor \\
L13 & Maigiginya & 52.80 & Marginal \\
L14 & Romi & 52.62 & Marginal \\
L15 & Railway Bridge & 54.04 & Marginal \\
\hline WQI = Water Quality Index & &
\end{tabular}

The WQI values of all the sampling locations shown in Table 14 were used in mapping the entire sampled portion of the river via Inverse Distance Weighted (IDW) interpolation method as could be seen in Figure 2.

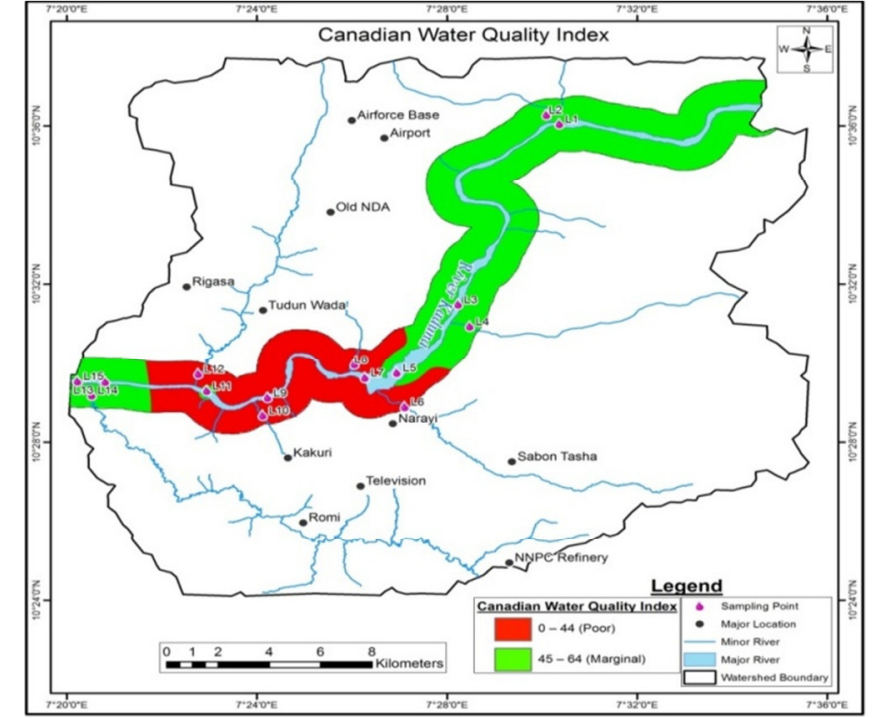

Fig 2: Canadian WQI map of River Kaduna

Table 14 divulge that among the 15 sampling locations, only locations L6 (Kutimbi), L8 (Kigo), L10 (Breweries) and L12 (Rigasa) recorded poor WQI. This could be attributed to the anthropogenic 
activities within the areas draining to these locations. However, Figure 2 revealed that the communities and towns draining to these sampling locations are Kakuri, Narayi, Sabon Tasha, Tudun Wada and Rigasa. These communities or towns are majorly industrialized and built-up areas within the watershed. The WQI map (Figure 2) also indicates that the water quality of the river upstream of Narayi community was marginal based on the Canadian WQI. Nevertheless, the quality of the river became poor in between Narayi and Rigasa communities and thereafter, the water quality started improving downstream at a point in between River Rigasa and River Romi.

Conclusion: Based on the results obtained in this research, it could be concluded that the WQI of River Kaduna on the Canadian scale is mostly marginal. However, the areas with high impairment level (poor) along the river are located within Sabon Tasha, Narayi, Kakuri, Tudun Wada and Rigasa communities. Hence, Regulatory agencies are advised to check the anthropogenic activities occurring within the watershed with more emphasis at Sabon Tasha, Narayi, Kakuri, Tudun Wada and Rigasa communities.

\section{REFERENCES}

Adebayo, OO (2014). Evaluation of Spatio-Temporal Characteristics of River Water Quality in OgunOsun River Basin in Nigeria Using Geographical Information System and Multivariate Techniques. A Ph.D Thesis submitted to the Post graduate School of University of Ibadan, Nigeria.

Adie, DB (2008). Multivariate Statistical Analysis of Pollution Characteristics in Rivers of the Middle Galma Basin, Nigeria. A Ph.D Dissertation submitted to the Post graduate School of Ahmadu Bello University, Zaria, Nigeria.
APHA (2005). Standard Methods for Examination of Water and Waste Water. American Public Health Association, Washington, DC.

Carlos, M; Alejandra, VV (2014). Journal of Urban and Environmental Engineering, Vol. 1, No.3, pp18-25.

CCME (2001). Canadian Council of Ministers of Environment: Water Quality Index User's Manual. Canadian Water Quality Guidelines for the Protection of Aquatic Life, pp 1-5.

Ekiye, E; Luo, Z (2010). Water Quality Monitoring in Nigeria; Case Study of Nigeria's Industrial Cities. Journal of American Science, 6(4), pp 2228.

Esengul, K; Cem, T; Arzu, C (2014). Monitoring Stream Water Quality: A Statistical Evaluation. Poland Journal of Environmental Studies. Vol. 23, No. 5, pp 1637-1647.

ESRI, (2015). ArcGIS 10.5: Using ArcGIS Spatial Analyst. Software User Guide ESRI, USA.

Mohammed, AA (2013). Water Quality Study of River Kaduna. International Journal of Advanced Research Volume 1, Issue 7, pp 467474

Mohammed, AE; Ibrahim, I; Badaru, YU; Garba, IK; Kudu, D (2015). Assessment of Effluents Discharged From Textile Industries in Selected Villages in Kaduna State, Nigeria. African Journal of Environmental Science and Technology, Vol. 9(5), pp 385-389.

Yusuf, RO; Durojaiye, AO; Salawudeen, TA (2008). Pollution Monitoring Along Kaduna River. International Journal of Environmental Science, Vol. 4, No. 4, pp 76-82 